\title{
Common fixed point theorems for compatible mappings of type (A) satisfying certain contractive conditions in partial metric space
}

\author{
Durdana Lateef
}

College of Science, Taibah University, Al-Madinah Al-Munawwarah, 41411, Kingdom of Saudi Arabia.

\begin{abstract}
The aim of this paper is to prove common fixed point theorems for compatible mappings of type (A) for three self-mappings satisfying certain contractive conditions and its topological properties in partial metric spaces.
\end{abstract}

Keywords: Fixed point, self-mappings, compatibility of type (A), partial metric space.

2010 MSC: 47H10, 54H 25.

(C)2019 All rights reserved.

\section{Introduction and preliminaries}

Matthews [8] introduced the notion of partial metric space and extended the Banach contraction principle to the class of complete partial metric space. After remarkable contribution of Matthews, many authors have studied partial metric space and its topological properties. Matthews discussed not only the general topological properties of partial metric spaces, but also some properties of convergence of sequences. The concept of compatible mapping was introduced by Jungck in the year 1986 [4] and proved that weakly commuting mappings are compatible mappings. In 1993 Cho et al. [5] introduced compatible of type (A) and proved common fixed point. In this paper we obtained common fixed points of contractive type self-mappings on partial metric spaces which cannot be deduced from the corresponding results in metric spaces. An example is also established to show that the result is a real generalization of analogous results for metric spaces.

We begin with some basic known definitions and lemmas on partial metric space which will be used in the sequel. Throughout this article, $N, \mathrm{R}^{+}$, and $\mathrm{R}$ denote the set of natural numbers, the set of positive real numbers, and the set of real numbers, respectively.

Before proceeding further, we recall some relevant concepts.

Email address: drdurdanamaths@gmail.com (Durdana Lateef)

doi: $10.22436 /$ jnsa.012.01.04 
Definition 1.1. A pair $(S, T)$ of self-mappings of a metric space $(X, d)$ is said to be compatible mappings, if

$$
\lim _{n \rightarrow \infty} d\left(S T x_{n}, T S x_{n}\right)=0,
$$

whenever $\left\{x_{n}\right\}$ is a sequence in $X$ such that

$$
\lim _{n \rightarrow \infty} S x_{n}=\lim _{n \rightarrow \infty} T x_{n}=t
$$

for some $t \in X$.

In 1993 Jungck et al. [5] introduced the notion of compatible mappings of type (A) in such a way.

Definition 1.2. A pair $(S, T)$ of self-mappings of a metric space $(X, d)$ is said to be compatible mappings of type (A), if

$$
\lim _{n \rightarrow \infty} d\left(S T x_{n}, T T S x_{n}\right)=0 \text { and } \lim _{n \rightarrow \infty} d\left(T S x_{n}, T T S x_{n}\right)=0,
$$

whenever $\left\{x_{n}\right\}$ is a sequence in $X$ such that

$$
\lim _{n \rightarrow \infty} S x_{n}=\lim _{n \rightarrow \infty} T x_{n}=t
$$

for some $t \in X$.

Matthews [8] gave the concept of partial metric space in this way.

Definition 1.3. A partial metric on a nonempty set $X$ is a function $p: X \times X \rightarrow[0, \infty)$ such that for all $x, y, z \in X:$

$\left(P_{1}\right) p(x, x)=p(y, y)=p(x, y)$ if and only if $x=y$;

$\left(P_{2}\right) p(x, x) \leqslant p(x, y)$;

$\left(P_{3}\right) p(x, y)=p(y, x)$;

$\left(P_{4}\right) p(x, z) \leqslant p(x, y)+p(y, z)-p(y, y)$.

The pair $(X, p)$ is then called a partial metric space. Also, each partial metric $p$ on $X$ generates a $T_{0}$ topology $\tau_{p}$ on $X$ with a base of the family of open p-balls $\left\{B_{p}(x, r): x \in X, r>0\right\}$, where $B_{p}(x, r)=$ $\{y \in X: p(x, y)<p(x, x)+r\}$. If $(X, p)$ is a partial metric space, then the function $p^{s}: X \times X \rightarrow R^{+}$given by $p^{s}(x, y)=2 p(x, y)-p(x, x)-p(y, y), x, y \in X$, is a metric on $X$. A basic example of a partial metric space is the pair $\left(R^{+}, p\right)$, where $p(x, y)=\max \{x, y\}$ for all $x, y \in R^{+}$.

Lemma 1.4 ([8]). Let $(X, p)$ be a partial metric space, then we have the following.

1. A sequence $\left\{x_{n}\right\}$ in a partial metric space $(X, p)$ converges to a point $x \in X$ if and only if

$$
\lim _{n \rightarrow \infty} p\left(x, x_{n}\right)=p(x, x) .
$$

2. A sequence $\left\{x_{n}\right\}$ in a partial metric space $(X, p)$ is called a Cauchy sequence if the $\lim _{n, m \rightarrow \infty} p\left(x_{n}, x_{m}\right)$ exists and is finite.

3. A partial metric space $(X, p)$ is said to be complete if every Cauchy sequence $\left\{x_{n}\right\}$ in $X$ converges to a point $x \in X$, that is $p(x, x)=\lim _{n, m \rightarrow \infty}\left(x_{n}, x_{m}\right)$.

4. A partial metric space $(\mathrm{X}, \mathrm{p})$ is complete if and only if the metric space $\left(\mathrm{X}, \mathrm{p}^{\mathrm{s}}\right)$ is complete. Furthermore, $\lim _{n \rightarrow \infty} p^{s}\left(x_{n}, z\right)=0$ if and only if $p(z, z)=\lim _{n \rightarrow \infty} p\left(x_{n}, z\right)=\lim _{n, m \rightarrow \infty} p\left(x_{n}, x_{m}\right)$.

\section{Main results}

Now I state my main result.

Theorem 2.1. Let $(\mathrm{X}, \mathrm{p})$ be a complete partial metric space and $\mathrm{S}, \mathrm{T}, \mathrm{f}: \mathrm{X} \rightarrow \mathrm{X}$ be self-mappings satisfying the following assertions:

(i) $S(X) \cup T(X) \subseteq f(X)$; 
(ii)

$$
p(S x, T y) \leqslant a \max \{p(f x, f y), p(f x, S x), p(f y, T y)\}+b\{p(f x, T y)+p(f y, S x)\}
$$

for all $x, y \in X$ and $a, b>0$ with $a+2 b<1$;

(iii) one of the pairs $(\mathrm{S}, \mathrm{f})$ or $(\mathrm{T}, \mathrm{f})$ is compatible of type (A);

(iv) the mapping $f$ is continuous.

Then the mappings $\mathrm{S}, \mathrm{T}$, and $\mathrm{f}$ have a unique common fixed point.

Proof. Let $x_{0}$ be an arbitrary point of $X$. We define the sequence $\left\{f x_{n}\right\}$ by

$$
f x_{2 n+1}=S x_{2 n} \text { and } f x_{2 n+2}=T x_{2 n+1}
$$

for all $n=0,1,2, \ldots$. From the inequality (2.1), we have

$$
\begin{aligned}
p\left(f x_{2 n+1}, f x_{2 n+2}\right)= & p\left(S x_{2 n}, T x_{2 n+1}\right) \\
\leqslant & a \max \left\{p\left(f x_{2 n}, f x_{2 n+1}\right), p\left(f x_{2 n}, S x_{2 n}\right), p\left(f x_{2 n+1}, T x_{2 n+1}\right)\right\} \\
& +b\left\{p\left(f x_{2 n}, T x_{2 n+1}\right)+p\left(f x_{2 n+1}, S x_{2 n}\right)\right\} \\
= & a \max \left\{p\left(f x_{2 n}, f x_{2 n+1}\right), p\left(f x_{2 n}, f x_{2 n+1}\right), p\left(f x_{2 n+1}, f x_{2 n+2}\right)\right\} \\
& +b\left\{p\left(f x_{2 n}, f x_{2 n+2}\right)+p\left(f x_{2 n+1}, f x_{2 n+1}\right)\right\} .
\end{aligned}
$$

By using (P4), we get

$$
\begin{aligned}
p\left(f x_{2 n+1}, f x_{2 n+2}\right) \leqslant & a \max \left\{p\left(f x_{2 n}, f x_{2 n+1}\right), p\left(f x_{2 n+1}, f x_{2 n+2}\right)\right\} \\
& +b\left\{p\left(f x_{2 n}, f x_{2 n+1}\right)+p\left(f x_{2 n+1}, f x_{2 n+2}\right)\right\}-p\left(f x_{2 n+1}, f x_{2 n+1}\right),
\end{aligned}
$$

which can written as

$$
p\left(f x_{2 n+1}, f x_{2 n+2}\right) \leqslant a \max \left\{p\left(f x_{2 n}, f x_{2 n+1}\right), p\left(f x_{2 n+1}, f x_{2 n+2}\right)\right\}+b\left\{p\left(f x_{2 n}, f x_{2 n+1}\right)+p\left(f x_{2 n+1}, f x_{2 n+2}\right)\right\} .
$$

Now two cases arise:

Case 1. If $\max \left\{p\left(f x_{2 n}, f x_{2 n+1}\right), p\left(f x_{2 n+1}, f x_{2 n+2}\right)\right\}=p\left(f x_{2 n}, f x_{2 n+1}\right)$, then from previous inequality, we get

$$
p\left(f x_{2 n+1}, f x_{2 n+2}\right) \leqslant a p\left(f x_{2 n}, f x_{2 n+1}\right)+b\left\{p\left(f x_{2 n}, f x_{2 n+1}\right)+p\left(f x_{2 n+1}, f x_{2 n+2}\right)\right\},
$$

which can be written in simplifying form as

$$
p\left(f x_{2 n+1}, f x_{2 n+2}\right) \leqslant \frac{(a+b)}{(1-b)} p\left(f x_{2 n}, f x_{2 n+1}\right) .
$$

Let $\frac{a-b}{1-b}=\lambda_{1}$, then from above we have

$$
p\left(f x_{2 n+1}, f x_{2 n+2}\right) \leqslant \lambda_{1} p\left(f x_{2 n}, f x_{2 n+1}\right) .
$$

Case 2. If $\max \left\{p\left(f x_{2 n}, f x_{2 n+1}\right), p\left(f x_{2 n+1}, f x_{2 n+2}\right)\right\}=p\left(f x_{2 n+1}, f x_{2 n+2}\right)$, then from previous inequality, we get

$$
p\left(f x_{2 n+1}, f x_{2 n+2}\right) \leqslant a p\left(f x_{2 n+1}, f x_{2 n+2}\right)+b\left\{p\left(f x_{2 n}, f x_{2 n+1}\right)+p\left(f x_{2 n+1}, f x_{2 n+2}\right)\right\},
$$

which can be written in simplifying form as

$$
p\left(f x_{2 n+1}, f x_{2 n+2}\right) \leqslant \frac{b}{(1-a-b)} p\left(f x_{2 n}, f x_{2 n+1}\right) .
$$


Let $\frac{\mathrm{b}}{1-\mathrm{a}-\mathrm{b}}=\lambda_{2}$ then from above we have

$$
p\left(f x_{2 n+1}, f x_{2 n+2}\right) \leqslant \lambda_{2} p\left(f x_{2 n}, f x_{2 n+1}\right) .
$$

As $a+2 b<1$, so we will have both $\lambda_{1}$ and $\lambda_{2}$ less than 1 . If we take $\lambda=\max \left\{\lambda_{1}, \lambda_{2}\right\}$, then from both cases we have

$$
p\left(f x_{2 n+1}, f x_{2 n+2}\right) \leqslant \lambda p\left(f x_{2 n}, f x_{2 n+1}\right) .
$$

If we repeat the same procedure as above, we get

$$
p\left(f x_{2 n}, f x_{2 n+1}\right) \leqslant \lambda p\left(f x_{2 n-1}, f x_{2 n}\right) .
$$

Inductively we have

$$
p\left(f x_{2 n+1}, f x_{2 n+2}\right) \leqslant \lambda^{2 n+1} p\left(f x_{0}, f x_{1}\right) .
$$

Hence

$$
p\left(f x_{n}, f x_{n+1}\right) \leqslant \lambda^{n} p\left(f x_{0}, f x_{1}\right) .
$$

Now we prove that the sequence $\left\{f x_{n}\right\}$ is a Cauchy sequence in $(X, p)$.

Let $m, n \in N$, with $m>n$ and consider

$$
\begin{aligned}
p\left(f x_{n}, f x_{n+m}\right) \leqslant & p\left(f x_{n}, f x_{n+1}\right)+p\left(f x_{n+1}, f x_{n+2}\right)+\cdots+p\left(f x_{n+m-1}, f x_{n+m}\right) \\
& \left.-p\left(f x_{n+}, f x_{n+1}\right)+p\left(f x_{n+2}, f x_{n+2}\right)+\cdots+p\left(f x_{n+m-1}, f x_{n+m-1}\right)\right) .
\end{aligned}
$$

By using the inequality (2.2), we get

$$
p\left(f x_{n}, f x_{n+m}\right) \leqslant\left[\lambda^{n}+\lambda^{n+1}+\cdots+\lambda^{n+m-1}\right] p\left(f x_{0}, f x_{1}\right) \leqslant \frac{\lambda^{n}}{1-\lambda} p\left(f x_{0}, f x_{1}\right) .
$$

As $\lambda<1$, so we have $p\left(f x_{n}, f x_{n}+m\right) \rightarrow 0$ as $m, n \rightarrow \infty$. Hence

$$
\lim _{n, m \rightarrow \infty} p\left(f x_{n}, f x_{n+m}\right)=0 .
$$

We have the following relation

$$
p^{s}(x, y)=2 p(x, y)-p(x, x)-p(y, y)
$$

So we can write it as

$$
p^{s}\left(f x_{n}, f x_{n+m}\right)=2\left(f x_{n}, f x_{n+m}\right)-\left(f x_{n}, f x_{n}\right)-\left(f x_{n+m}, f x_{n+m}\right) \leqslant 2\left(f x_{n}, f x_{n+m}\right) .
$$

Applying (2.3), we have

$$
\lim _{n, m \rightarrow \infty} p^{s}\left(f x_{n}, f x_{n+m}\right)=0
$$

Hence the sequence $\left\{f x_{n}\right\}$ is a Cauchy sequence in $\left(X, p^{s}\right)$. Since $(X, p)$ is complete, so the corresponding metric space $\left(X, p^{s}\right)$ is also complete. Therefore, the sequence $\left\{f x_{n}\right\}$ converges to some $z \in X$ with respect to the metric $p$ that is

$$
\lim _{n \rightarrow \infty} p^{s}\left(f x_{n}, z\right)=0
$$

Since

$$
p\left(f x_{n}, f x_{n}\right) \leqslant p\left(f x_{n}, f x_{n+1}\right) \leqslant \lambda^{n} p\left(f x_{0}, f x_{1}\right) \rightarrow 0 \text { as } n \rightarrow \infty .
$$

Therefore

$$
p(z, z) \leqslant \lim _{n \rightarrow \infty} p\left(f x_{n}, z\right) \leqslant \lim _{n, m \rightarrow \infty} p\left(f x_{n}, f x_{n+m}\right)=0
$$


Thus the sequence $\left\{f x_{n}\right\}$ is a Cauchy sequence in $(X, p)$. Since $(X, p)$ is complete, so there exists some $z \in X$ such that $\lim _{n \rightarrow \infty} f x_{n}=z$. It follows that the sequences $\left\{S x_{n}\right\}$ and $\left\{T x_{n+1}\right\}$ also converge to $z$. First we suppose that the pair $(f, S)$ is compatible of type (A). Then from the inequality (2.1), we have

$$
\begin{gathered}
p\left(S f x_{2 n}, T x_{2 n+1}\right) \leqslant a \max \left\{p\left(f f x_{2 n}, f x_{2 n+1}\right), p\left(f f x_{2 n}, S f x_{2 n}\right), p\left(f x_{2 n+1}, T x_{2 n+1}\right)\right\} \\
+b\left\{p\left(f f x_{2 n}, T x_{2 n+1}\right)+p\left(f x_{2 n+1}, S f x_{2 n}\right)\right\} .
\end{gathered}
$$

Since the mapping $f$ is continuous, so we have $f f x_{2 n} \rightarrow f z$ and $f S x_{2 n} \rightarrow f z$ as $n \rightarrow \infty$. From the supposition that the pair $(f, S)$ is compatible of type $(A)$, we have

$$
\mathrm{fS} x_{2 n} \rightarrow f z, S f x_{2 n} \rightarrow f z \text { and } f f x_{2 n} \rightarrow f z
$$

as $n \rightarrow \infty$. So letting $n \rightarrow \infty$ in inequality (2.4), we have

$$
p(f z, z) \leqslant a \max \{p(f z, z), p(f z, f z), p(z, z)\}+b\{p(f z, z)+p(z, f z)\} .
$$

As $p(x, x) \leqslant p(x, y)$, so we can write

$$
p(f z, z) \leqslant a \max \{p(f z, z), p(f z, z), p(f z, z)\}+b\{p(f z, z)+p(z, f z)\} .
$$

Thus after simplifying, we have

$$
(1-a-2 b) p(f z, z) \leqslant 0
$$

It follows that $f z=z$.

Now from inequality (2.1), we have

$$
p\left(S z, T x_{2 n+1}\right) \leqslant a \max \left\{p\left(f z, f x_{2 n+1}\right), p(f z, S z), p\left(f x_{2 n+1}, T x_{2 n+1}\right)\right\}+b\left\{p\left(f z, T x_{2 n+1}\right)+p\left(f x_{2 n+1}, S z\right)\right\} .
$$

Letting $n \rightarrow \infty$, we have

$$
p(S z, z) \leqslant a \max \{p(f z, z), p(f z, S z), p(z, z)\}+b\{p(f z, z)+p(z, S z)\} .
$$

As $f z=z$, so one can easily get $S z=z$ from above. Similarly, we consider

$$
p\left(S x_{2 n}, T z\right) \leqslant a \max \left\{p\left(f x_{2 n}, f z\right), p\left(f x_{2 n}, S x_{2 n}\right), p(f z, T z)\right\}+b\left\{p\left(f x_{2 n}, T z\right)+p\left(f z, S x_{2 n}\right)\right\} .
$$

Letting $n \rightarrow \infty$, we have

$$
p(z, T z) \leqslant a \max \{p(z, f z), p(z, z), p(f z, T z)\}+b\{p(z, T z)+p(f z, z)\} .
$$

Using the fact as $f z=z$, we can obtain $T z=z$. Thus we have $S z=T z=f z=z$ that is $z$ is a common fixed point of $S, T$, and $f$. Similarly we can prove that $z$ is a common fixed point of $S, T$, and $f$ when the pair $(f, T)$ is compatible of type $(A)$.

Now we prove the uniqueness of this theorem. Let $w$ be another common fixed point of $S, T$ and $f$ other than $z$. Then $S w=\mathrm{T} w=\mathrm{f} w=w$ and $S z=\mathrm{T} z=\mathrm{f} z=z$ but $w=z$. Now from the inequality (2.1), we have

$$
\begin{aligned}
p(z, w)=p(S z, T w) & \leqslant a \max \{p(f z, f w), p(f z, S z), p(f w, T w)\}+b\{p(f z, T w)+p(f w, S z)\} \\
& =\operatorname{a~max}\{p(z, w), p(z, z), p(w, w)\}+b\{p(z, w)+p(w, z)\} \\
& =a p(z, w)+2 b p(z, w)=(a+2 b) p(z, w)
\end{aligned}
$$

which is a contradiction to the fact that $z=w$. Thus $z=w$. This completes the proof.

Corollary 2.2. Let $(X, p)$ be a partial metric space and $S, f: X \rightarrow X$ be self-mappings satisfying the following assertions: 
(i) $S(X) \subseteq f(X)$;

(ii)

$$
p(S x, S y) \leqslant a \max \{p(f x, f y), p(f x, S x), p(f y, S y)\}+b\{p(f x, S y)+p(f y, S x)\}
$$

for all $x, y \in X$ and $a, b>0$ with $a+2 b<1$;

(iii) the pair $(\mathrm{S}, \mathrm{f})$ is compatible of type $(A)$;

(iv) the mapping $f$ is continuous.

Then the mappings $\mathrm{S}$ and $\mathrm{f}$ have a unique common fixed point.

Remark 2.3. By taking different mappings as Identity mapping in my main result we can get variety of corollaries.

Theorem 2.4. Let $(X, p)$ be a complete partial metric space and $S, T, f: X \rightarrow X$ be self-mappings satisfying the following assertions:

(i) $S(X) \cup T(X) \subseteq f(X)$;

(ii)

$$
p(S x, T y) \leqslant b\{p(f x, T y)+p(f y, S x)\}
$$

for all $\mathrm{x}, \mathrm{y} \in \mathrm{X}$ and $0 \leqslant \mathrm{~b}<\frac{1}{2}$

(iii) one of the pairs $(S, f)$ or $(T, f)$ is compatible of type $(A)$;

(iv) the mapping $f$ is continuous.

Then the mappings $S, T$ and $f$ have a unique common fixed point.

\section{Common fixed point results for F-contraction}

F-contraction is recent development in the field of fixed point theory and has lot of generalizations in current research. This concept was given by Wardowski [9] in 2012. We begin this section with the following basic definition of F-contraction.

Definition 3.1. Let $F: R^{+} \rightarrow R$ be a mapping satisfying the following conditions:

$\left(\mathrm{F}_{1}\right) \mathrm{F}$ is strictly increasing;

$\left(F_{2}\right)$ for all sequence $\alpha_{n} \subseteq R^{+}, \lim _{n \rightarrow \infty} \alpha_{n}=0$ if and only if $\lim _{n \rightarrow \infty} F\left(\alpha_{n}\right)=-\infty$;

$\left(F_{3}\right)$ there exists $0<k<1$ such that $\lim _{n \rightarrow 0^{+}} \alpha^{k} F(\alpha)=0$.

A mapping $T: X \rightarrow X$ is said to be an F-contraction if there exists $\tau \in R^{+}$such that for all $x, y \in X$,

$$
d(T x, T y)>0=\Rightarrow \tau+F \cdot d(T x, T y) \leqslant F \cdot d(x, y) .
$$

To be consistent with Wardowski [9], we denote the set of all functions $F: R^{+} \rightarrow R$ by J, satisfying the above conditions. Later on many authors generalized this result in a different way in various generalized metric spaces. For more details in this direction I refer the reader to $[1-3,6,7,10]$.

In this paper, I establish a common fixed point theorem for three self-mappings in the setting of complete partial metric spaces and obtained different results as corollaries of my main result. I also give a remark with a suitable example that my result cannot be derived from the ordinary metric space.

Now I state and prove my main result for three self-mappings in partial metric space.

Theorem 3.2. Let $(\mathrm{X}, \mathrm{p})$ be a complete partial metric space and $\mathrm{S}, \mathrm{T}, \mathrm{f}: \mathrm{X} \rightarrow \mathrm{X}$ be self-mappings satisfying the following assertions:

1. $S(X) \cup T(X) \subseteq f(X)$; 
2. $p(S x, T y)>0$ implies

$$
\tau+F(p(S x, T y)) \leqslant F(p(f x, f y))
$$

for all $\mathrm{x}, \mathrm{y} \in \mathrm{X}$ and $\mathrm{F} \in \mathrm{J}$;

3. one of the pairs $(S, f)$ or $(T, f)$ is compatible of type $(A)$;

4. the mapping $f$ is continuous.

Then $\mathrm{S}, \mathrm{T}$, and $\mathrm{f}$ have a unique common fixed point.

Proof. Let $x_{0}$ be an arbitrary point of X. As I have done in previous theorem, I define the sequence $\left\{f x_{n}\right\}$ by

$$
f x_{2 n+1}=S x_{2 n} \text { and } f x_{2 n+2}=T x_{2 n+1}
$$

for all $n=0,1,2, \ldots$. From the inequality (3.1), we have

$$
\tau+F\left(p\left(f x_{2 n+1}, f x_{2 n+2}\right)\right)=\tau+F\left(p\left(S x_{2 n}, T x_{2 n+1}\right)\right) \leqslant F\left(p\left(f x_{2 n}, f x_{2 n+1}\right)\right),
$$

which implies that

$$
F\left(p\left(f x_{2 n+1}, f x_{2 n+2}\right)\right) \leqslant F\left(p\left(f x_{2 n}, f x_{2 n+1}\right)\right)-\tau .
$$

From (3.1) we have

$$
\begin{aligned}
\tau+F\left(p\left(f x_{2 n+2}, f x_{2 n+3}\right)\right) & =\tau+F\left(p\left(T x_{2 n+1}, S x_{2 n+2}\right)\right) \\
& =\tau+F\left(p\left(S x_{2 n+2}, T x_{2 n+1}\right)\right) \leqslant F\left(p\left(f x_{2 n+2}, f x_{2 n+1}\right)\right)=F\left(p\left(f x_{2 n+1}, f x_{2 n+2}\right)\right),
\end{aligned}
$$

which implies that

$$
F\left(p\left(f x_{2 n+2}, f x_{2 n+3}\right)\right) \leqslant F\left(p\left(f x_{2 n+1}, f x_{2 n+2}\right)\right)-\tau .
$$

Thus for all $n=1,2, \ldots$

$$
F\left(p\left(f x_{n}, f x_{n+1}\right)\right) \leqslant F\left(p\left(f x_{n-1}, f x_{n}\right)\right) \tau \leqslant F\left(p\left(f x_{n-2}, f x_{n-1}\right)\right)-2 \tau \leqslant \cdots \leqslant F\left(p\left(f x_{0}, f x_{1}\right)\right)-n \tau
$$

for all $n \in N$. Since $F \in J$, so by taking limit as $n \rightarrow \infty$ in (3.2),

$$
\lim _{n \rightarrow \infty} F\left(p\left(f x_{n}, f x_{n+1}\right)\right)=-\infty \Leftarrow \Rightarrow \lim _{n \rightarrow \infty} p\left(f x_{n}, f x_{n+1}\right)=0 .
$$

Now from $\left(\mathrm{F}_{3}\right)$, there exists $0<k<1$ such that,

$$
\lim _{n \rightarrow \infty}\left[p\left(f x_{n}, f x_{n+1}\right)\right]^{k} F p\left(f x_{n}, f x_{n+1}\right)=0 .
$$

By (3.4), we have

$$
\begin{aligned}
& p\left(f x_{n}, f x_{n+1}\right)^{k} F\left(p\left(f x_{n}, f x_{n+1}\right)\right)-p\left(f x_{n}, f x_{n+1}\right)^{k} F\left(p\left(f x_{0}, f x_{1}\right)\right) \\
& \left.\quad<p\left(f x_{n}, f x_{n+1}\right)^{k}\left[F p\left(f x_{0}, f x_{1}\right)-n \tau\right)-F\left(p\left(f x_{0}, f x_{1}\right)\right)\right]=-n \tau\left[p\left(f x_{n}, f x_{n+1}\right)\right]^{k} \leqslant 0 .
\end{aligned}
$$

By taking limit as $n \rightarrow \infty$ in (3.5) and applying (3.3) and (3.4),

$$
\lim _{n \rightarrow \infty} n\left[p\left(f x_{n}, f x_{n+1}\right)\right]^{k}=0 .
$$

It follows from (3.6) that there exists, $n_{1} \in N$ such that,

$$
n\left[p\left(f x_{n}, f x_{n+1}\right)\right]^{k} \leqslant 1
$$


for all $n>n_{1}$. This implies,

$$
p\left(f x_{n}, f x_{n+1}\right) \leqslant \frac{1}{n^{\frac{1}{k}}} .
$$

Now we prove that $\left\{x_{n}\right\}$ is a Cauchy sequence.

For $m>n>n_{1}$ we have,

$$
p\left(f x_{n}, f x_{n+m}\right) \leqslant \sum_{i=n}^{n+m-1} p\left(f x_{i}, f x_{n+m}\right) \leqslant \sum_{i=n}^{n+m-1} \frac{1}{i^{\frac{1}{k}}} .
$$

Since, $0<k<1$, then $\sum_{i=1}^{\infty} \frac{1}{i \frac{1}{k}}$ converges, so we have $p\left(f x_{n}, f x_{n+m}\right) \rightarrow 0$ as $m, n \rightarrow \infty$. Hence

$$
\lim _{n, m \rightarrow \infty} p\left(f x_{n}, f x_{n+m}\right)=0 .
$$

As we have the following relation

$$
p^{s}(x, y)=2 p(x, y)-p(x, x)-p(y, y) .
$$

So we can write it as

$$
p^{s}\left(f x_{n}, f x_{n+m}\right)=2\left(f x_{n}, f x_{n+m}\right)-\left(f x_{n}, f x_{n}\right)-\left(f x_{n+m}, f x_{n+m}\right) \leqslant 2\left(f x_{n}, f x_{n+m}\right) .
$$

Applying (3.7), we have

$$
\lim _{n, m \rightarrow \infty} p^{s}\left(f x_{n}, f x_{n+m}\right)=0 .
$$

Hence the sequence $\left\{f x_{n}\right\}$ is a Cauchy sequence in $\left(X, p^{s}\right)$. Since $(X, p)$ is complete, so the corresponding metric space $\left(X, p^{s}\right)$ is also complete. Therefore, the sequence $\left\{f x_{n}\right\}$ converges to some $z \in X$ with respect to the metric $p^{s}$, that is

$$
\lim _{n \rightarrow \infty} p^{s}\left(f x_{n}, z\right)=0
$$

Since

$$
p\left(f x_{n}, f x_{n}\right) \leqslant p\left(f x_{n}, f x_{n+1}\right) \leqslant n 1 / k \rightarrow 0 \text { as } n \rightarrow \infty .
$$

Therefore

$$
p(z, z) \leqslant \lim _{n \rightarrow \infty} p\left(f x_{n}, z\right) \leqslant \lim _{n, m \rightarrow \infty} p\left(f x_{n}, f x_{n+m}\right)=0 .
$$

Thus the sequence $\left\{f x_{n}\right\}$ is a Cauchy sequence in $(X, p)$. Since $(X, p)$ is complete, so there exists some $z \in X$ such that $\lim _{n \rightarrow \infty} f x_{n}=z$. It follows that the sequences $\left\{S x_{n}\right\}$ and $\left\{T x_{n+1}\right\}$ also converge to $z$. First we suppose that the pair $(f, S)$ is compatible of type (A) and contrary suppose that $f z \neq z$. Then from the inequality (3.1),

$$
\tau+F\left(p\left(S f x_{2 n}, T x_{2 n+1}\right)\right) \leqslant F\left(p\left(f f x_{2 n}, f x_{2 n+1}\right)\right) .
$$

As $F$ is strictly increasing, so we get

$$
p\left(S f x_{2 n}, T x_{2 n+1}\right) \leqslant p\left(f f x_{2 n}, f x_{2 n+1}\right) .
$$

Since the mapping $f$ is continuous, so we have $f f x_{2 n} \rightarrow f z$ and $f S x_{2 n} \rightarrow f z$ as $n \rightarrow \infty$. From the supposition that the pair $(f, S)$ is compatible of type $(A)$, we have

$$
\mathrm{fS} \mathrm{x}_{2 \mathrm{n}} \rightarrow \mathrm{f} z, \mathrm{Sf} \mathrm{x}_{2 \mathrm{n}} \rightarrow \mathrm{f} z \text { and } \mathrm{ffx} \mathrm{x}_{2 \mathrm{n}} \rightarrow \mathrm{fz}
$$

as $n \rightarrow \infty$. So letting $n \rightarrow \infty$ in inequality (3.8), we have

$$
p(f z, z)<p(f z, z),
$$


which is a contradiction. Thus $f z=z$.

Now from the inequality (3.1), we have

$$
\tau+F\left(p\left(S z, T x_{2 n+1}\right)\right) \leqslant F\left(p\left(f z, f x_{2 n+1}\right)\right) .
$$

As $F$ is strictly increasing, so we get

$$
p\left(S z, T x_{2 n+1}\right) \leqslant p\left(f z, f x_{2 n+1}\right) .
$$

Letting $n \rightarrow \infty$, we have

$$
p(S z, z) \leqslant p(f z, z)
$$

As $f z=z$, so one can easily get $S z=z$ from above. Similarly, we consider

$$
\tau+F\left(p\left(S x_{2 n}, T z\right)\right) \leqslant F\left(p\left(f x_{2 n}, f z\right)\right) .
$$

As $F$ is strictly increasing, so we get

$$
p\left(S x_{2 n}, T z\right) \leqslant p\left(f x_{2 n}, f z\right) .
$$

Letting $n \rightarrow \infty$, we have

$$
p(z, T z) \leqslant p(z, f z)
$$

Using the fact as $f z=z$, we can obtain $T z=z$. Thus we have $S z=T z=f z=z$ that is $z$ is a common fixed point of $S, T$ and $f$. Similarly we can prove that $z$ is a common fixed point of $S, T$, and $f$ when the pair $(f, T)$ is compatible of type $(A)$.

Now we prove the uniqueness of this theorem. Let $w$ be another common fixed point of $S, T$, and $f$ other than $z$. Then $S w=T w=f w=w$ and $S z=T z=f z=z$ but $w=z$. Now from the inequality (3.1), we have

$$
\tau+\mathrm{F}(\mathrm{p}(z, w))=\tau+\mathrm{F}(\mathrm{p}(\mathrm{S} z, \mathrm{~T} w)) \leqslant \mathrm{F}(\mathrm{p}(\mathrm{f} z, \mathrm{f} w))=\mathrm{F}(\mathrm{p}(z, w)),
$$

which is a contradiction to the fact that $z \neq w$, because $\tau>0$. Thus $z=w$. This completes the proof.

Corollary 3.3. Let $(X, p)$ be a complete partial metric space and $S, T: X \rightarrow X$ be self-mappings satisfying the following assertion:

$$
p(S x, T y)>0 \text { implies } \tau+F(p(S x, T y)) \leqslant F(p(x, y))
$$

for all $\mathrm{x}, \mathrm{y} \in \mathrm{X}$ and $\mathrm{F} \in \mathrm{J}$. Then $\mathrm{S}$ and $\mathrm{T}$ have a unique common fixed point.

Proof. Taking $\mathrm{f}=\mathrm{I}$ (identity mapping).

Corollary 3.4. Let $(X, p)$ be a complete partial metric space and $S, T: X \rightarrow X$ be self-mappings satisfying the following assertion:

$$
p(S x, T y) \leqslant \lambda p(x, y)
$$

for all $\mathrm{x}, \mathrm{y} \in \mathrm{X}$ where $0 \leqslant \lambda<1$. Then $\mathrm{S}$ and $\mathrm{T}$ have a unique common fixed point.

Remark 3.5. The above theorem cannot be deduced from similar result of metric spaces. Actually the contractive condition (3.9) for a pair $S, T: X \rightarrow X$ of mappings on a metric space $(X, d)$ that is

$$
d(S x, T y) \leqslant \lambda d(x, y) \text { for all } x, y \in X
$$

is not attainable. Because $S \neq T$ implies that $S v \neq T v$ for some $v \in X$, then $d(S v, T v)>0=\lambda d(v, v)$.

Condition (3.9) is not satisfied for $x=y=v$. However the same condition in partial metric space is feasible to find common fixed point result for a pair of mappings. This fact can be seen again in the following example. 
Example 3.6. Let $X=[0,1]$ and $p(x, y)=\max \{x, y\}$ and $S, T: X \rightarrow X$ be defined by

$$
\mathrm{Sx}=\frac{1}{8} \mathrm{x}, \mathrm{T} x=\frac{3}{8} \mathrm{x}
$$

Then

$$
d(S x, T y)=\max \left\{\frac{1}{8} x, \frac{3}{8} x\right\} \quad \text { and } \quad d(S x, T y)=\frac{1}{8} \max \{x, 3 y\} \leqslant \frac{5}{11} \max \{x, y\} \leqslant \lambda p(x, y) .
$$

Therefore, for $\lambda=\frac{3}{10}$ all the conditions of Corollary 3.4 are satisfied to find common fixed point of $S$ and $\mathrm{T}$. However, note that for any metric $\mathrm{d}$ on $\mathrm{X}$

$$
\mathrm{d}(\mathrm{S} 1, \mathrm{~T} 1)=\mathrm{d}\left(\frac{1}{8}, \frac{3}{8}\right)>\lambda \mathrm{d}(1,1)=0 \text { for any } \lambda \in[0,1) .
$$

Therefore common fixed points of $S$ and $T$ cannot be obtained from a corresponding metric fixed point theorem.

\section{References}

[1] J. Ahmad, A. Al-Rawashdeh, A. Azam, New fixed point theorems for generalized F-contractions in complete metric spaces, Fixed Point Theory Appl., 2015 (2015), 18 pages. 3

[2] L. A. Alnaser, D. Lateef, J. Ahmad, Some new fixed point theorems for compatible mappings in partial metric spaces, J. Math. Computer Sci., 18 (2018), 346-356.

[3] N. Hussain, J. Ahmad, L. Ciric, A. Azam, Coincidence point theorems for generalized contractions with application to integral equations, Fixed Point Theory Appl., 2015 (2015), 13 pages. 3

[4] G. Jungck, Compatible mappings and common fixed points, Internat. J. Math. Math. Sci., 9 (1986), 771-779. 1

[5] G. Jungck, P. P. Murthy, Y. J. Cho, Compatible mappings of type (A) and common fixed points, Math. Japon., 38 (1993), 381-390. 1, 1

[6] D. Lateef, Fixed Point Theorem of Nonlinear Contraction in Metric Space, Adv. Fixed Point Theory, 8 (2018), $247-254$. 3

[7] D. Lateef, J. Ahmad, A. E. Al-Mazrooei, Common Fixed Point Theorems For Generalized Contractions, J. Math. Anal., 8 (2017), 157-166. 3

[8] S. G. Matthews, Partial Metric Topology, in: Papers on general topology and applications, 1994 (1994), 183-197. 1, $1,1.4$

[9] D. Wardowski, Fixed points of a new type of contractive mappings in complete metric spaces, Fixed Point Theory Appl., 2012 (2012), 6 pages. 3, 3

[10] D. Wardowski, N. Van Dung, Fixed points of F-weak contractions on complete metric space, Demonstr. Math., 47 (2014), 146-155. 3 